\title{
Homoclinic classes with shadowing
}

\author{
Jiweon Ahn' ${ }^{1}$, Keonhee Lee ${ }^{1}$ and Manseob Lee ${ }^{2^{*}}$
}

\footnotetext{
* Correspondence:

Imsds@mokwon.ac.kr

${ }^{2}$ Department of Mathematics,

Mokwon University, Daejeon, 302-

729, Korea

Full list of author information is

available at the end of the article
}

\begin{abstract}
We show that for $C^{1}$ generic diffeomorphisms, an isolated homoclinic class is shadow-able if and only if it is a hyperbolic basic set.

Mathematics Subject Classification 2000: 37C20; 37C05; 37C29; $37 D 05$.

Keywords: shadowing property, basic set, hyperbolic, generically, homoclinic class
\end{abstract}

\section{Introduction}

Let $M$ be a closed $C^{\infty}$ manifold, and denote by $d$ the distance on $M$ induced from the Riemannian metric $\|\cdot\|$ on the tangent bundle TM. Denote by $\operatorname{Diff}(M)$ the space of diffeo-morphisms of $M$ endowed with the $C^{1}$-topology. Let $f \in \operatorname{Diff}(M)$. For $\delta>0$, a sequence of points $\left\{x_{i}\right\}_{i=a}^{b}(-\infty \leq a<b \leq \infty)$ in $M$ is called a $\delta$-pseudo orbit of $f$ if $d(f$ $\left.\left(x_{i}\right), x_{i+1}\right)<\delta$ for all $a \leq i \leq b-1$. A closed $f$-invariant set $\Lambda \subset M$ is said to be chain transitive if for any points $x, y \leadsto \Lambda$ and $\delta>0$, there is a $\delta$-pseudo orbit $\left\{x_{i}\right\}_{i=a_{\delta}}^{b \delta} \subset \Lambda\left(a_{\delta}\right)<b_{\delta}$ of $f$ such that $x_{a_{\delta}}=x$ and $x_{b_{\delta}}=y$. For given $x, y \in M$, we write $x$ $\rightsquigarrow y$ if for any $\delta>0$, there is a $\delta$-pseudo orbit $\left\{x_{i}\right\}_{i=a}^{b}(a<b)$ of $f$ such that $x_{a}=x$ and $x_{b}=y$. Write $x \nabla y$ if $x \rightsquigarrow y$ and $y \rightsquigarrow x$. The set of points $\{x \in M: x \otimes x\}$ is called the chain recurrent set of $f$ and is denoted by $\mathcal{R}(f)$. If we denote the set of periodic points $f$ by $P(f)$, then $P(f) \subset \Omega(f) \subset \mathcal{R}(f)$, where $\Omega(f)$ is the non-wandering set of $f$. The relation $\otimes$ on $\mathcal{R}(f)$ induces an equivalence relation, whose classes are called chain components of $f$. Every chain component of $f$ is a closed $f$-invariant set.

Denote by $\left.f\right|_{\Lambda}$ the restriction of $f$ to the set $\Lambda$. We say that $\left.f\right|_{\Lambda}$ has the shadowing property (or, $\Lambda$ is shadowable for $f$ ) if for any $\epsilon>0$ there is $\delta>0$ such that for any $\delta$ pseudo orbit $\left\{x_{i}\right\}_{i \in \mathbb{Z}} \subset \Lambda$ of $f$ there is $y \in M$ such that $d\left(f^{\prime}(y), x_{i}\right)<\epsilon$, for $i \in \mathbb{Z}$.

It is well known that if $p$ is a hyperbolic periodic point $f$ with period $k$ then the sets

$$
\begin{gathered}
W^{s}(p)=\left\{x \in M: f^{k n}(x) \rightarrow p \text { as } n \rightarrow \infty\right\} \text { and } \\
W^{u}(p)=\left\{x \in M: f^{-k n}(x) \rightarrow p \text { as } n \rightarrow \infty\right\}
\end{gathered}
$$

are $C^{1}$-injectively immersed submanifolds of $M$. Every point in the transversal intersection $\left(W^{s}(p) \pitchfork W^{u}(p)\right)$ of $W^{S}(p)$ and $W^{u}(p)$ is called the homoclinic point of $f$ associated to $p$. The closure of the homoclinic points of $f$ associated to $p$ is called the homoclinic class of $f$ and it is denoted by $H_{f}(p)$.

Note that the homoclinic class $H_{f}(p)$ is a subset of the chain component $C_{f}(p)$ of $f$ containing $p$. We consider only the hyperbolic periodic orbits of saddle type. We say

(c) 2012 Ahn et al; licensee Springer. This is an Open Access article distributed under the terms of the Creative Commons Attribution License (http://creativecommons.org/licenses/by/2.0), which permits unrestricted use, distribution, and reproduction in any medium, provided the original work is properly cited. 
that two hyperbolic periodic points $p$ and $q$ are homoclinically related, and write $p \sim q$, if $W^{s}(p) \pitchfork W^{u}(q) \neq \emptyset$ and $W^{u}(p) \pitchfork W^{s}(q) \neq \emptyset$. We know that if $p \sim q$ then index $(p)=$ index $(q)$. Here index $(p)$ denotes the dimension of the stable manifold $W^{s}(p)$ of $p$. By Smale's transverse homoclinic theorem, we know that the closure of the set of homoclinically related points with a hyperbolic periodic point $p$ is the homoclinic class $H_{f}(p)$ of $f$ associated to $p$.

We say that $\Lambda$ is hyperbolic if the tangent bundle $T_{\Lambda} M$ has a $D f$-invariant splitting $E^{s}$ $\oplus E^{u}$ and there exist constants $C>0$ and $0<\lambda<1$ such that

$$
\left\|\left.D_{x} f^{n}\right|_{E_{x}^{s}}\right\| \leq C \lambda^{n} \text { and }\left\|\left.D_{x} f^{-n}\right|_{E_{x}^{u}}\right\| \leq C \lambda^{n}
$$

for all $x \in \Lambda$ and $n \geq 0$. It is well-known that if $\Lambda$ is hyperbolic, then $\Lambda$ is shadowable.

We say that $\Lambda$ is isolated (or locally maximal) if there is a compact neighborhood $U$ of $\Lambda$ such that $n_{n \in Z} f^{\prime}(U)=\Lambda$. We say that a subset $\mathcal{G} \subset \operatorname{Diff}(M)$ is residual if $\mathcal{G}$ contains the intersection of a countable family of open and dense subsets of Diff $(M)$; in this case, $\mathcal{G}$ is dense in $\operatorname{Diff}(M)$. A property "P" is said to be $\left(C^{1}\right)$-generic if "P" holds for all diffeo-morphisms which belong to a residual subset of $\operatorname{Diff}(M)$. We use the terminology "for $C^{1}$ generic $f^{\prime}$ to express "there is a residual subset $\mathcal{G} \subset \operatorname{Diff}(M)$ such that for any $f \in \mathcal{G}$...".

In [1], Abdenur and Díaz posed the following conjecture:

Conjecture. For $C^{1}$ generic $f, f$ is shadowable if and only if it is hyperbolic.

In this article, we gives a partial answer to the above conjecture. First, we show that $C^{1}$-generically, the chain recurrent set is hyperbolic if and only if it has the shadowing property. Next, we prove that $C^{1}$-generically, the isolated homoclinic class containing a hyperbolic periodic point is shadowable if and only if it is hyperbolic.

It is explain in [2] that every $C^{1}$-generic diffeomorphism come in one of two types: tame diffeomorphisms, which have a finite number of homoclinic classes and whose nonwandering sets admits partitions into a finite number of disjoint transitive sets; and wild diffeomor-phisms, which have an infinite number of homoclinic classes and whose nonwandering sets admit no such partitions. It is easy to show that if a diffeomorphism has a finite number of chain components, then every chain component is locally maximal, and therefore, every chain component of a tame diffeomorphism is locally maximal. Hence, we can get the following result.

Theorem 1.1 For $C^{1}$ generic $f$, if $f$ is tame then the following two conditions are equivalent:

(a) $\mathcal{R}(f)$ is hyperbolic,

(b) $\mathcal{R}(f)$ is shadowable.

We say that a closed $f$-invariant set $\Lambda$ is basic, if $\Lambda$ is isolated, $\left.f\right|_{\Lambda}$ is transitive and the periodic orbits are dense in $\Lambda$. The main result of this article is the following.

Theorem 1.2 For $C^{1}$ generic $f$, the isolated homoclinic class $H_{f}(p)$ of $f$ containing a hy-perbolic periodic point $p$ is shadowable if and only if it is a hyperbolic basic set. 
A similar result for locally maximal chain transitive sets was proved in [3]. More precisely, it is proved that $C^{1}$-generically, every locally maximal chain transitive set is hyperbolic if it is shadowable.

\section{Proof of Theorem 1.2}

Let $M$ and $f \in \operatorname{Diff}(M)$ be as before. In this section, to prove Theorem 1.2, we use the techniques developed by Mañé [4]. Let $\Lambda_{j}(f)$ be the closure of the set of hyperbolic periodic points of $f$ with index $j(0 \leq j \leq \operatorname{dim} M)$. If there is a $C^{1}$-neighborhood $\mathcal{U}(f)$ of $f$ such that for any $g \in \mathcal{U}(f)$, any periodic points of $g$ are hyperbolic, then $f$ satisfies both Axiom A and the no-cycle condition. To prove our result, we first note that if $p$ is homoclinically related to $q$, then $H_{f}(p)=H_{f}(q)$.

Lemma 2.1 Suppose that $f$ has the shadowing property on $H_{f}(p)$. Then for any hyperbolic periodic point $q \in H_{f}(p), W^{s}(p) \cap W^{u}(q) \neq \emptyset$, and $W^{u}(p) \cap W^{s}(q) \neq \emptyset$.

Proof. We will only show that $W^{s}(p) \cap W^{u}(q) \neq \emptyset$. Since $p$ and $q$ are hyperbolic saddles, there are $\epsilon(p)>0$ and $\epsilon(q)>0$ such that

- both $W_{\epsilon(p)}^{s}(p)$ and $W_{\epsilon(q)}^{u}(q)$ are $C^{1}$-embedded disks,

- if $d\left(f^{n}(x), f^{n}(p)\right)<\epsilon(p)$ for $n \geq 0$, then $x \in W_{\epsilon(p 1)}^{s}(p)$,

- if $d\left(f^{p}(x), f^{n}(q)\right)<\epsilon(q)$ for $n \leq 0$ then $x \in W_{\epsilon(q)}^{u}(q)$.

Set $\epsilon=\min \{\epsilon(p), \epsilon(q)\}$, and let $0<\delta=\delta(\epsilon)<\epsilon$ be a number which satisfies the shadowing property of $\left.f\right|_{H_{f}(p)}$ with respect to $\epsilon$. To simplify, we assume that $f(p)=p$ and $f(q)=q$. Since $H_{f}(p)=\overline{\{q \in P(f): q \sim p\}}$, we can choose a hyperbolic periodic point $\gamma$ such that $\gamma$ is homoclinically related to $p$, and $d(q, \gamma)<\delta / 2$. For $x \in W^{s}(p) \pitchfork W^{u}(\gamma)$, choose $n_{1}>0$ and $n_{2}>0$ such that

$$
d\left(f^{n_{1}}(x), p\right)<\delta / 4 \text { and } d\left(f^{-n_{2}}(x), \gamma\right)<\delta / 4 .
$$

Thus

$$
d\left(f^{-n_{2}}(x), q\right)<d\left(f^{-n_{2}}(x), \gamma\right)+d(\gamma, q)<\delta / 2 .
$$

Therefore, we can construct a $\delta$-pseudo orbit;

$$
\xi=\left\{\ldots, q, f^{-n_{2}+1}(x), \ldots, f^{-1}(x), x, f(x), \ldots, f^{n_{1}-1}(x), p, \ldots,\right\} .
$$

Then we have $\xi \subset H_{f}(p)$. Since $f$ has the shadowing property on $H_{f}(p)$, there is a point $y \in M$ such that $d\left(\dot{f}(y), x_{i}\right)<\epsilon$, for $i \in \mathbb{Z}$. Thus

$$
f^{n_{1}+l}(\gamma) \in W_{\epsilon}^{s}(p) \text { and } f^{-n_{2}-l}(\gamma) \in W_{\epsilon}^{u}(q), \quad \text { for } l>0 .
$$

Therefore, $y \in f^{-n_{1}-l}\left(W_{\epsilon}^{s}(p)\right) \cap f^{n_{2}+l}\left(W_{\epsilon}^{u}(q)\right)$. This means $y \in W^{s}(p) \cap W^{u}(q)$, and so $W^{s}(p) \cap W^{u}(q) \neq \emptyset$.

Lemma 2.2 There is a residual set $\mathcal{G}_{1} \subset \operatorname{Diff}(M)$ such that $f \in \mathcal{G}_{1}$ satisfies the following properties: 
(a) Every periodic point of $f$ is hyperbolic and all their invariant manifolds are intersect transversely (Kupka-Smale).

(b) $C_{f}(p)=H_{f}(p)$, where $p$ is a hyperbolic periodic point $([5])$.

Lemma 2.3 There is a residual set $\mathcal{G}_{2} \subset \operatorname{Diff}(M)$ such that if $f \in \mathcal{G}_{2}$, and $f$ has the shadowing property on $H_{f}(p)$, then for any hyperbolic periodic point $q \in H_{f}(p)$,

$$
W^{s}(p) \pitchfork W^{u}(q) \neq \emptyset, \quad \text { and } W^{u}(p) \pitchfork W^{s}(q) \neq \emptyset .
$$

Proof. Let $\mathcal{G}_{2}$ be as in Lemma 2.2(a), and let $f \in \mathcal{G}_{2}$. Take a hyperbolic saddle point $q \in H_{f}(p) \cap P(f)$. Since $f$ has the shadowing property on $H_{f}(p)$, we know that $W^{s}(p) \cap W^{u}(q) \neq \emptyset$ and $W^{u}(p) \cap W^{s}(q) \neq \emptyset$ by Lemma 2.1. Since $f \in \mathcal{G}_{2}$, we know that

$$
W^{s}(p) \pitchfork W^{u}(q) \neq \emptyset, \quad \text { and } W^{u}(p) \pitchfork W^{s}(q) \neq \emptyset .
$$

Proposition 2.4 For $C^{1}$ generic $f$, if the homoclinic class $H_{f}(p)$ is isolated and shadowable, then there exist constants $m>0$ and $0<\lambda<1$ such that for any periodic point $q$ $\in H_{f}(p), \operatorname{index}(q)=\operatorname{dim} W^{\varsigma}(q)$,

$$
\prod_{i=0}^{\pi(q)-1}\left\|\left.D f^{m}\right|_{E^{s}\left(f^{i m}(q)\right)}\right\|<\lambda^{\pi(q)}, \prod_{i=0}^{\pi(q)-1}\left\|\left.D f^{m}\right|_{\left.E^{s} f^{i m}(q)\right)}\right\|<\lambda^{\pi(q)}
$$

and

$$
\left\|\left.D f^{m}\right|_{E^{s}(q)}\right\| \cdot\left\|\left.D f^{-m}\right|_{E^{u}\left(f^{m}(q)\right)}\right\|<\lambda^{2},
$$

where $\pi(q)$ denotes the period of $q$.

We introduce the following notion which was introduced in [6]. For $\eta>0$ and $f \in$ $\operatorname{Diff}(M)$, a $C^{1}$ curve $\gamma$ is called an $\eta$-simply periodic curve of $f$ if

- $\gamma$ is diffeomorphic to $[0,1]$ and its two end points are hyperbolic periodic points of $f$,

- $\gamma$ is periodic with period $\pi(\gamma)$, i.e., $f^{\tau(\gamma)}(\gamma)=\gamma$, and $l\left(f^{\dot{ }}(\gamma)\right)<\eta$ for any $0 \leq i \leq \pi(\gamma)$

- 1, where $l(\gamma)$ denotes the length of $\gamma$.

- $\gamma$ is normally hyperbolic.

Let $p$ be a periodic point of $f$. For $\delta \in(0,1)$, we say $p$ has a $\delta$-weak eigenvalue if D $f^{\pi(p)}(p)$ has an eigenvalue $\mu$ such that $(1-\delta)^{\pi(p)}<\mu<(1+\delta)^{\pi(p)}$.

Lemma 2.5 [6] There is a residual set $\mathcal{G}_{3} \subset \operatorname{Diff}(M)$ such that for any $f \in \mathcal{G}_{3}$, any hyper-bolic periodic point $p$ of $f$, and

(a) for any $\eta>0$, if for any $C^{1}$ neighborhood $\mathcal{U}(f)$ of $f$, some $g \in \mathcal{U}(f)$ has an $\eta$ simply periodic curve $\gamma$ such that two endpoints of $\gamma$ are homoclinic related with $p_{g}$, then $f$ has an $2 \eta$-simply periodic curve $\alpha$ such that two endpoints of $\alpha$ are homoclinically related with $p$;

(b) for any $\delta>0$, if $f$ has a periodic point $q \sim p$ with $\delta$-weak eigenvalue, then $f$ has $a$ periodic point $q$ ' $\sim p$ with $\delta$-weak eigenvalue, whose eigenvalues are all real. 
The following lemma shows that the map $f \mapsto C_{f}(p)$ is upper semi-continuous.

Lemma 2.6 For any $\epsilon>0$, there is $\delta>0$ such that if $d_{1}(f, g)<\delta$ then $C_{g}\left(p_{g}\right) \subset B_{\epsilon}\left(C_{f}\right.$ $(p))$, where $d_{1}$ denotes the $C^{1}$-metric on $\operatorname{Diff}(\mathrm{M})$.

Proof. See [[7], Lemma].

Let $H_{f}(p)$ be the homoclinic class of $f$ associated to $p$. It is known that the map $f \mapsto$ $H_{f}(p)$ is lower semi-continuous. Thus by Lemma $2.3(\mathrm{~b})$, there is a residual set $\mathcal{R}$ in $\operatorname{Diff}(M)$ such that for any $f$ in $\mathcal{R}$, the map $f \mapsto H_{f}(p)\left(=C_{f}(p)\right)$ is semi-continuous.

Remark 2.7 There is a residual set $\mathcal{G}_{4} \subset \operatorname{Diff}(M)$ such that for any $f \in \mathcal{G}_{4}$, we have the following property. Let $C_{f}(p)$ be the isolated chain component of $f$ containing $p$ in an open set $U$ in $M$. If $C_{f}(p)$ is semi-continuous, then for any $\epsilon>0$, there is $\delta>0$ such that if $d_{1}(f, g)<\delta$ then $C_{g}\left(p_{g}\right) \subset B_{\epsilon}\left(C_{f}(p)\right)$ and $C_{f}(p) \subset B_{\epsilon}\left(C_{g}\left(p_{g}\right)\right)$, where $d_{1}$ is the $C^{1}$ metric on $M$.

Let $p$ be a hyperbolic periodic point $f \in \operatorname{Diff}(M)$.

Remark 2.8 [6] There is a residual set $\mathcal{G}_{5} \subset \operatorname{Diff}(M)$ such that for any $f \in \mathcal{G}_{5}$ and any $\delta>0$, if every periodic point $q \sim p$ has no $2 \delta$-weak eigenvalue, then there is a $C^{1}$ neighborhood $\mathcal{U}(f)$ of $f$ such that for any $g \in \mathcal{U}(f)$ every periodic point $q$ which is homoclinically related to $p_{g}$ has no $\delta$-weak eigenvalue, where $p_{g}$ is the continuation of $p$.

Lemma 2.9 There is a residual set $\mathcal{G}_{6} \subset \operatorname{Diff}(M)$ such that if $f \in \mathcal{G}_{6}$, and the isolated homoclinic class $H_{f}(p)$ is shadowable, then there is a $\delta>0$ such that every periodic point $q \in H_{f}(p)$ has no $\delta$-weak eigenvalue.

Proof. Let $\mathcal{G}_{6}=\bigcap_{i=1}^{5} \mathcal{G}_{i}$ and let $f \in \mathcal{G}_{6}$. Assume that $f$ has the shadowing property on the homoclinic class $H_{f}(p)$. Note that $H_{f}(p)=C_{f}(p)$. Suppose that for any $\delta>0$ there is a hyperbolic periodic point $q \in C_{f}(p)$ such that $q$ has a $\delta$-weak eigenvalue. Since $f$ has the shadowing property on $C_{f}(p)$, for the point $q \in C_{f}(p) \cap P(f)$, we see that $q \sim p$ by Lemma 2.3. Let $U$ be an isolated neighborhood of $C_{f}(p)$, and let $\eta>0$ be a sufficiently small constant such that $B_{2 \eta}\left(C_{f}(p)\right) \subset U$. Then for any $C^{1}$-neighborhood $\mathcal{U}(f)$ of $f$, there is $g \in \mathcal{U}(f)$ such that $g$ has an $\eta / 2$-simply periodic curve $\mathcal{I}_{q}$, whose endpoints are homoclinically related to $p_{g}$, and $\eta / 2$-simply periodic curve $\mathcal{I}_{q} \subset C_{g}(p g) \subset B_{2 \eta}\left(C_{f}(p)\right.$ ) (for more details, see Theorem B in [8]). Then we know that for some $l>0, \mathcal{I}_{q}$ is a $g^{l}$-invariant small curve containing $p_{g}$ ( $q$ is the center of $\mathcal{I}_{q}$ ), where $q \sim p_{g}$. By Lemma 2.5(a), for the above $\eta>0 f$ has a $\eta$-simply periodic curve $\mathcal{J}_{q}$ such that the endpoints of $\mathcal{J}_{q}$ are homoclinically related to $p$. Then we see that

$$
\mathcal{J}_{q} \subset B_{2 \eta}\left(C_{f}(p)\right) \subset U
$$

Then for some $l^{\prime}>0, \mathcal{J}_{p}$ is a $f^{\prime}$-invariant small curve center at $q$. To simplify, we denote $f^{\prime}$ by $f$. Since $C_{f}(p)$ is an isolated in $U$, we have

$$
\mathcal{J}_{q} \subset \bigcap_{n \in \mathbb{Z}} f^{n}\left(B_{2 \eta}\left(C_{f}(p)\right)\right) \subseteq \bigcap_{n \in \mathbb{Z}} f^{n}(U)=C_{f}(p) .
$$

Since $\mathcal{J}_{q}$ is a simple periodic curve of $f,\left.f\right|_{\mathcal{J}_{q}}$ is the identity map. Since $\mathrm{f}$ has the shadowing property on $C_{f}(p), f$ must have the shadowing property on $\mathcal{J}_{q}$. But it is a contradiction. Thus every periodic point $q \in H_{f}(p)$ has no $\delta$-weak eigenvalue. 
Proof of Proposition 2.4. Let $f \in \mathcal{G}_{6}$, and suppose that there is a nonhyperbolic periodic point $q \in H_{f}(p)$. Then $q$ has a $\delta$-weak eigenvalue. This contradicts Lemma 2.9, and com-pletes the proof of Proposition 2.4 by Mañé [4].

Proof of Theorem 1.2. Let $f \in \mathcal{G}_{6}$, and let $C_{f}(p)$ be isolated in an open set $U$. Assume that $f$ has the shadowing property on $C_{f}(p)$. Then $C_{f}(p)$ satisfy the assumptions of Propo-sition 2.4. Since $f$ has the shadowing property on $C_{f}(p), C_{f}(p)$ is hyperbolic by the main result in [9]. Consequently, we have proved that $C^{1}$-generically, $H_{f}(p)$ is a hyperbolic basic set.

\section{Acknowledgements}

We wish to thank the referee for carefully reading the manuscript and providing us many good suggestions. J. Ahn was supported by the BK21 Mathematics Vision 2013 Project. K. Lee was supported by National Research Foundation of Korea (NRF) grant funded by the Korea government (No. 2011-0015193). M. Lee was supported by Basic Science Research Program through the National Research Foundation of Korea (NRF) funded by the Ministry of Education, Science and Technology (No. 2011-0007649).

\section{Author details}

'Department of Mathematics, Chungnam National University, Daejeon, 305-764, Korea ²Department of Mathematics, Mokwon University, Daejeon, 302-729, Korea

\section{Authors' contributions}

All authors conceived of the study, participated in its design and coordination, drafted the manuscript, participated in the sequence alignment, and read and approved the final manuscript.

\section{Competing interests}

The authors declare that they have no competing interests.

Received: 10 November 2011 Accepted: 23 April 2012 Published: 23 April 2012

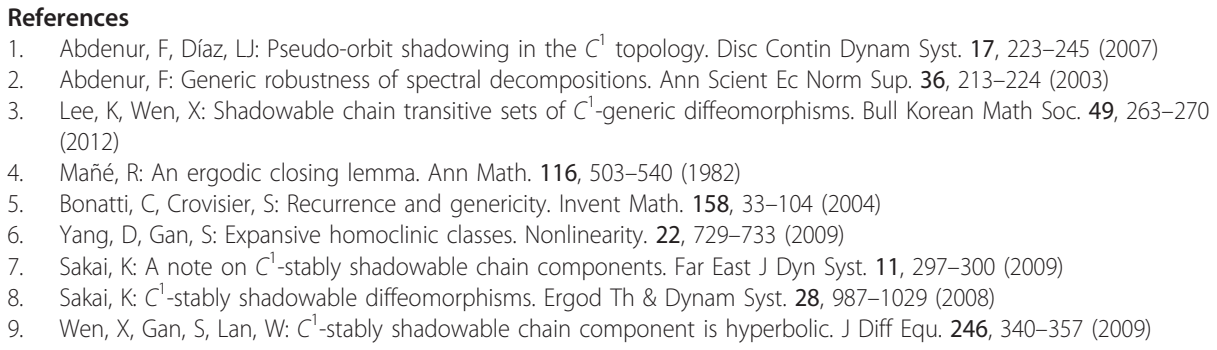

doi:10.1186/1029-242X-2012-97

Cite this article as: Ahn et al:: Homoclinic classes with shadowing. Journal of Inequalities and Applications 2012 2012:97.

\section{Submit your manuscript to a SpringerOpen ${ }^{\circ}$ journal and benefit from:}

- Convenient online submission

- Rigorous peer review

- Immediate publication on acceptance

- Open access: articles freely available online

- High visibility within the field

- Retaining the copyright to your article

Submit your next manuscript at $>$ springeropen.com 